\title{
Time-Domain Physical Optics Method for the Analysis of Wide-Band EM Scattering from Two-Dimensional Conducting Rough Surface
}

\author{
Jia Chungang, Guo Lixin, and Yang Pengju \\ School of Science, Xidian University, Xian 710071, China \\ Correspondence should be addressed to Jia Chungang; jcg.0502@163.com
}

Received 2 March 2013; Accepted 18 August 2013

Academic Editor: Daniel S. Weile

Copyright (c) 2013 Jia Chungang et al. This is an open access article distributed under the Creative Commons Attribution License, which permits unrestricted use, distribution, and reproduction in any medium, provided the original work is properly cited.

\begin{abstract}
Time-domain physical optics (TDPO) method is extended to investigate electromagnetic (EM) scattering from two-dimensional (2D) perfectly electrically conducting (PEC) rough surface in both time domain and frequency domain. The scheme requires relatively small amounts of computer memory and CPU time, and has advantage over the Kirchhoff Approximation (KA) method in obtaining transient response of rough surface by a program run. The 2D Gaussian randomly rough surface is generated by Monte Carlo method and then is partitioned into small triangle facets through the meshing preprocess. The accuracy of TDPO is validated by comparing the numerical results with those obtained by the KA method in both backward and specular directions. The transient response and its frequency distribution of radar cross section (RCS) from rough surface is shown, respectively. The scattering results from rough surface with different size in the specular direction are given. The influence of the root mean square height $(\sigma)$ and correlation length $(l)$ on electromagnetic scattering from PEC rough surface is discussed in detail. Finally, the comparisons of backscattering results at different incident angles are presented and analyzed.
\end{abstract}

\section{Introduction}

Nowadays, the problems of EM scattering from randomly rough surface have been widely applied in fields of remote sensing, target identification, radar detection, and so on. A lot of investigations are carried out to deal with this random problem in terms of roughness and slope of the rough surface. Take the approximate analytical methods, for example, the KA method [1], which is considerably valid approach to solve the problem that rough surface is relatively smooth, the small-perturbation method (SPM) in which standard derivation of rough surface is small enough compared with the wavelength [2], the small-slope approximation (SSA) that is applied to small and large surface roughness [3], and the two-scale method (TSM) [4], which considers that the rough surface can be separated into large- and smallscale surfaces, allowing use of physical optics (PO) and SPM methods. These techniques are limited by the roughness, the incident angle, and so forth. Some numerical methods are also employed, which include the Monte Carlo method [5], the finite difference time-domain (FDTD) $[6,7]$ method, method of moments (MOM) $[8,9]$, the finite element method (FEM) [10], and the forward back method (FBM) [11], the fast multipole method (FMM) [12].

However, these approaches and accelerated algorithms can hardly handle the model that is considerably electrically large due to complex computation. High frequency technologies are still extremely valid to solve the problem that the electrical size of rough surface is large, but high frequency methods in time domain rarely appeared in the previous literatures about investigating on scattering from the randomly rough surface, and transient scattering has important significance in synthetic aperture radar (SAR) imaging or inverse SAR (ISAR) imaging. Up to now, to our knowledge, few works have been reported for the wide-band scattering of rough surface by TDPO. In this paper, emphasis is put on discussing this situation in the time domain. The TDPO method is the time version of physical optics, which reserves the advantage of fast computing speed and less memory demand, and can obtain wide-band property by 
a program running. This paper is devoted to the wide-band scattering characteristics of the $2 \mathrm{D}$ rough surface by utilizing TDPO method, which can deal with the electrically large rough surface problem in the time domain. The earlier TDPO method is not carried out in the time domain directly. Each frequency component response is obtained by the frequency method, and the time-domain response of the origin signal is then achieved by inverse Fourier transform (IFT). TDPO is firstly presented by En-Yuan Sun, and the method is used to analyze the scattering field of paraboloidal reflector and hyperboloidal reflector [13]. In [14], the scheme is employed to deal with the large-size structure of the combinative objects.

In this paper, we employ TDPO to investigate scattering from the 2D PEC randomly rough surface. The method is performed to obtain the transient response reflected from rough surface, and then the property of wide-band frequency is obtained by fast Fourier transform (FFT). The formulation of TDPO is presented in [13], which derives the far field by transforming the equivalent electric current density and far-field expression in the frequency domain to those in the time domain. The model of $2 \mathrm{D}$ rough surface is presented by the Monte Carlo method. The comparison between the wide-band RCS obtained by TDPO and calculated by the sweep-frequency KA method verifies the validation of TDPO. Finally, the presented scheme is utilized to analyze scattering properties of 2D randomly rough surface.

The paper remaining is organized as follows. In Section 2, the formulation of TDPO is presented, as well as the theoretical formulae of $2 \mathrm{D}$ randomly rough surface which is of Gaussian type are given. Several examples of $2 \mathrm{D}$ rough surface generated with different parameters of root mean square height (rms height) and correlation length are demonstrated. Section 3 provides the validity of TDPO by comparing the numerical results with those obtained by sweep-frequency KA. The influence of size of rough surface, correlation length, rms height, and the incident angle on the scattering from rough surface is discussed. Finally, some concluding remarks are addressed in Section 4.

\section{Theory and Formulation}

2.1. Randomly Rough Surface Generation. Firstly, 2D randomly rough surface is modeled in order to determine its scattering characteristic. Using the spectrum density, rough surface is simulated by Monte Carlo method [15] by which the power spectrum is filtered in frequency domain, and then the height of rough surface can be obtained by inverse fast Fourier transform (IFFT). The height distribution function of rough surface is written as

$$
\begin{aligned}
f\left(x_{m}, y_{n}\right) \\
=\frac{1}{L_{x} L_{y}} \sum_{m_{k}=-M / 2+1}^{M / 2} \sum_{n_{k}=-N / 2+1}^{N / 2} F\left(k_{m_{k}}, k_{n_{k}}\right) \\
\times \exp \left[i\left(k_{m_{k}} x_{m}+k_{n_{k}} y_{n}\right)\right],
\end{aligned}
$$

where

$$
\begin{aligned}
F( & \left.k_{m_{k}}, k_{n_{k}}\right) \\
= & 2 \pi\left[L_{x} L_{y} S\left(k_{m_{k}}, k_{n_{k}}\right)\right]^{1 / 2} \\
& \cdot \begin{cases}\frac{[N(0,1)+i N(0,1)]}{\sqrt{2}}, & m_{k} \neq 0, \frac{M}{2}, \text { and } n_{k} \neq 0, \frac{N}{2} \\
N(0,1), & m_{k} \neq 0, \frac{M}{2}, \text { or } n_{k} \neq 0, \frac{N}{2},\end{cases}
\end{aligned}
$$

$L_{x}$ and $L_{y}$ are the lengths of the rough surface along the $x$-axis and $y$-axis, respectively. The numbers of discrete points are $M$ and $N . k_{m_{k}}$ and $k_{n_{k}}$ are the spatial frequency at the corresponding point. $N(0,1)$ is a random number following normal distributions function. $S\left(k_{m_{k}}, k_{n_{k}}\right)$ is the power spectrum density function, which is Gaussian type in this paper and is given as follows:

$$
S\left(k_{m_{k}}, k_{n_{k}}\right)=\sigma^{2} \frac{{ }^{2} l_{y}}{4 \pi} \exp \left(-\frac{k_{m_{k}}^{2} l_{x}^{2}+k_{n_{k}}^{2} l_{y}^{2}}{4}\right),
$$

where $\sigma$ is rms height, and $l_{x}$ and $l_{y}$ are the correlation lengths along the $x$ - and $y$-direction, by which the profile of the rough surface is determined. Examples of $2 \mathrm{D}$ randomly rough surfaces are simulated and depicted with different correlation length and rms height in Figures 1(a)-1(d).

\subsection{Time-Domain Physical Optics Method for 2D PEC Rough} Surface. Based on the generation of the rough surface profiles, the surface is divided into small triangle facets though mesh processing. For the conducting rough surface, the surface-current-density distribution by the physical optics method in the frequency domain approximates as [16]

$$
\vec{J}_{s}= \begin{cases}2 \widehat{n} \times \vec{H}^{\text {inc }}\left(\vec{r}^{\prime}, \omega\right), & \text { lit region } \\ 0, & \text { otherwise }\end{cases}
$$

where $\vec{H}^{\text {inc }}\left(\vec{r}^{\prime}, \omega\right)$ is the incident magnetic field, $\widehat{n}$ is the unit vector normal to the surface. The far scattering field in the frequency domain is approximately derived as

$$
\begin{aligned}
\vec{E}_{s}(\vec{r}, \omega)= & j k z_{0} \frac{\exp (-j k r)}{4 \pi r} \\
& \times \iint_{s} \widehat{r} \times\left[\widehat{r} \times \vec{J}_{s}\left(\vec{r}^{\prime}, \omega\right)\right] \exp \left(j \vec{k} \cdot \vec{r}^{\prime}\right) d s^{\prime} .
\end{aligned}
$$

In (5), $z_{0}$ is the characteristic impedance in free space. $\vec{r}$ is the distant observing point, and $\vec{r}^{\prime}$ is the scatterer integration point, where $\vec{J}_{s}\left(\vec{r}^{\prime}, \omega\right)$ is the surface current density. $s$ is the surface area of the lighten region. In order to derive the scattering field in the time domain, the IFT is needed for (5), and the electric field in time domain can be obtained as follows

$$
\vec{E}_{s}(\vec{r}, t)=\frac{\eta}{4 \pi r c} \iint_{s} \hat{r} \times\left[\widehat{r} \times \frac{\partial}{\partial t} \vec{J}_{s}\left(\vec{r}^{\prime}, t-\tau_{1}\right)\right] d s^{\prime},
$$

where $\tau_{1}=\vec{r} \cdot\left(\vec{r}-\vec{r}^{\prime}\right) / c$ is time delay from the integration point to the distant observing point. The relation of surface-current 


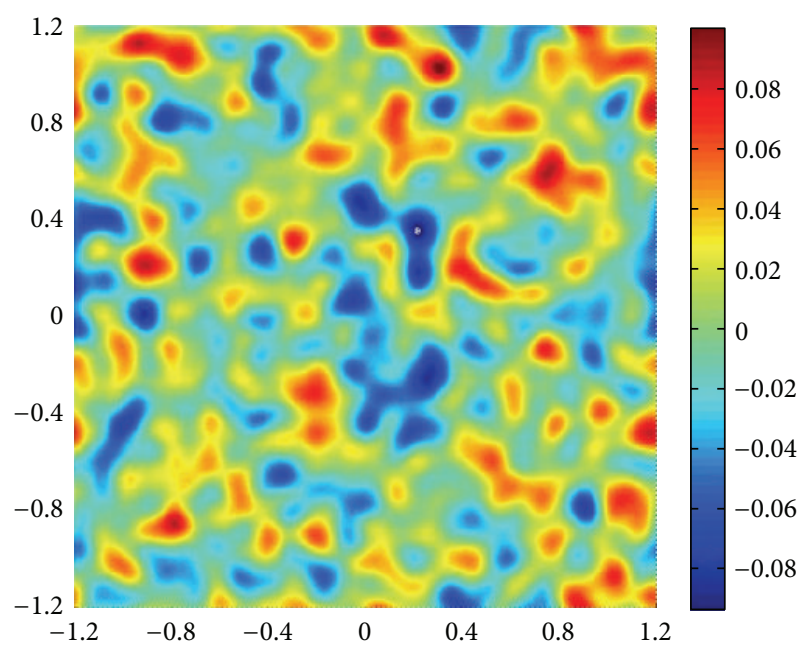

(a) $\sigma=0.03 \mathrm{~m}, l_{x}=l_{y}=0.1 \mathrm{~m}$

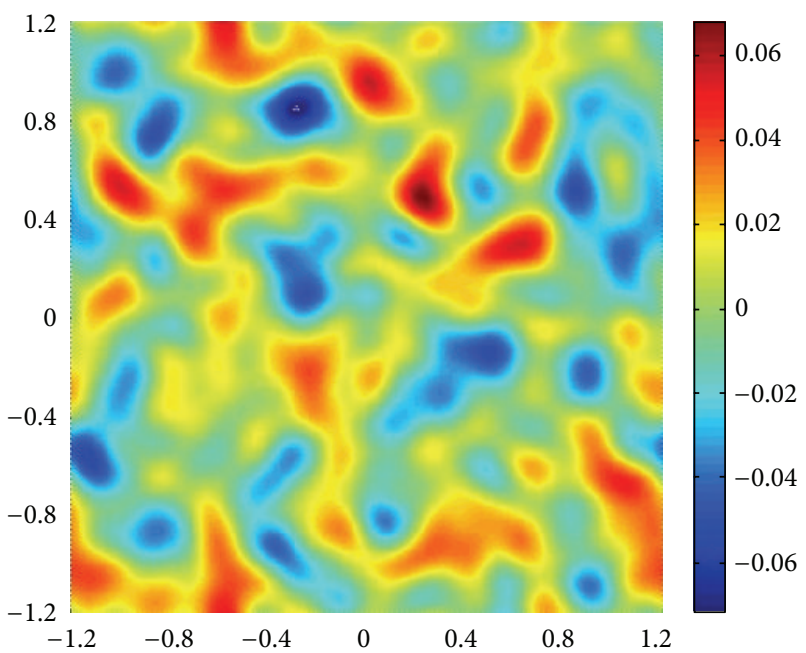

(c) $\sigma=0.02 \mathrm{~m}, l_{x}=l_{y}=0.15 \mathrm{~m}$

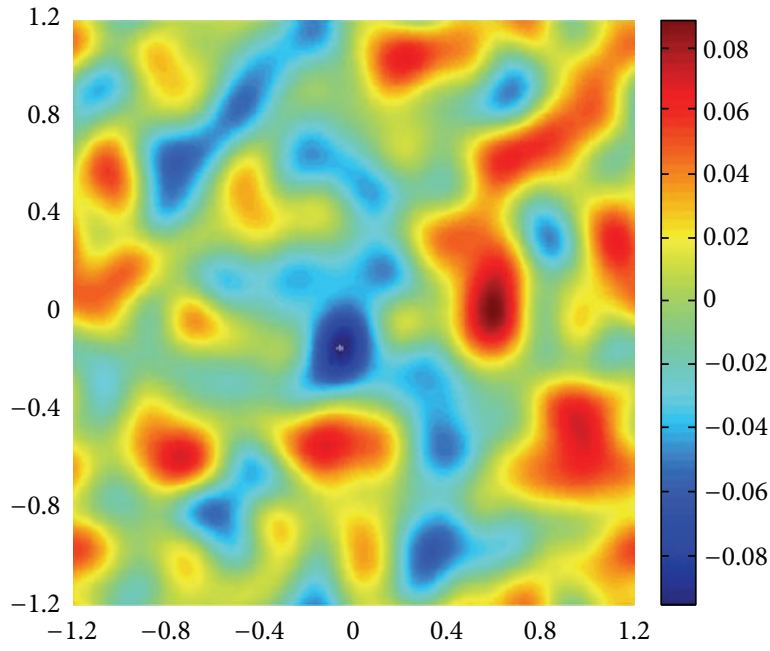

(b) $\sigma=0.03 \mathrm{~m}, l_{x}=l_{y}=0.2 \mathrm{~m}$

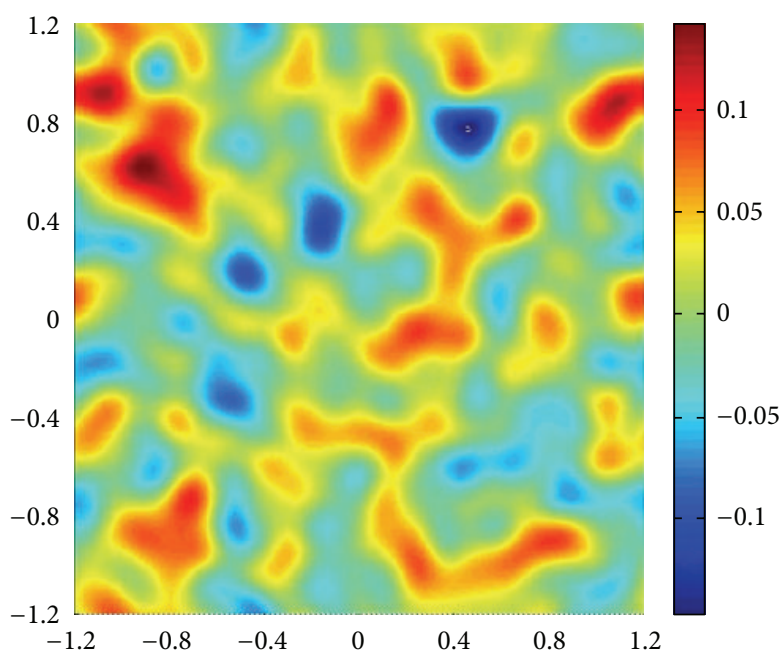

(d) $\sigma=0.04 \mathrm{~m}, l_{x}=l_{y}=0.15 \mathrm{~m}$

FIGURE 1: Simulation of 2D Gaussian randomly rough surface (the unit of bar is meter).

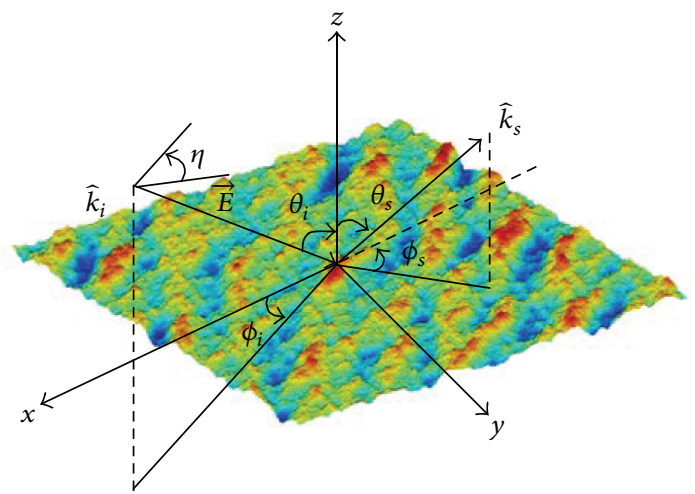

FIGURE 2: Definition of scattering geometry with relevant parameters in the coordinate $x y z$. 


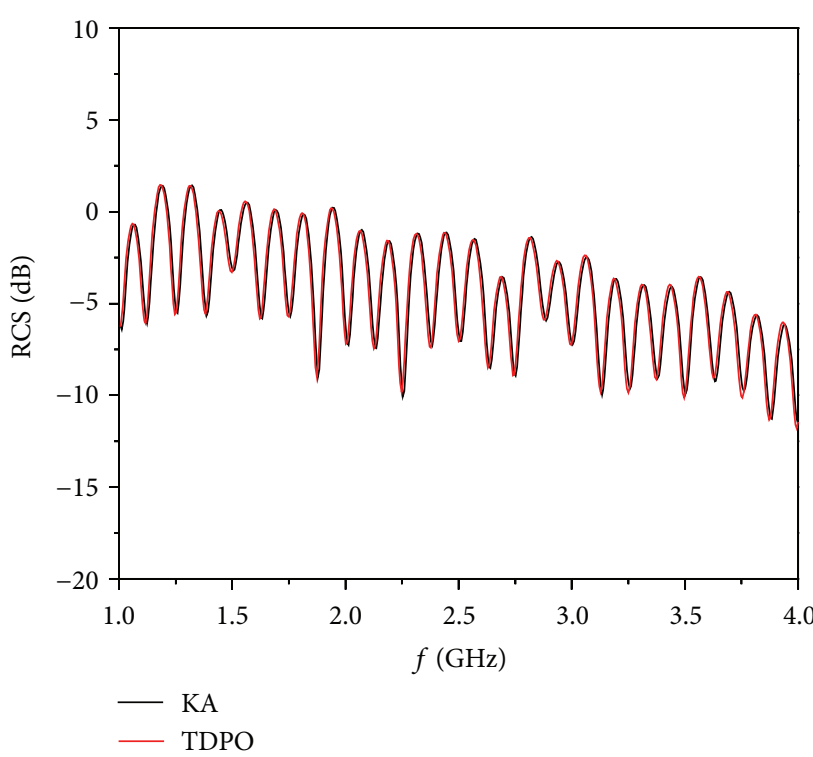

(a) $\theta \theta$ polarization in backward direction $\left(\theta_{i}=30^{\circ}\right)$

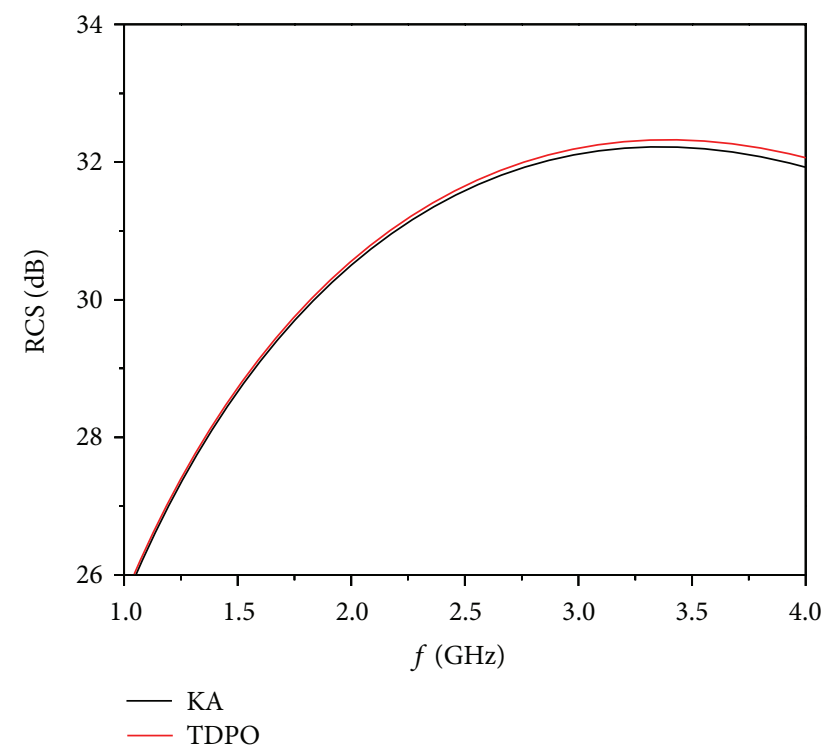

(b) $\varphi \varphi$ polarization in specular direction $\left(\theta_{i}=45^{\circ}\right)$

FIgURE 3: TDPO and KA methods for the wide-band RCS from 2D PEC rough surface.

density between the time domain and frequency domain satisfies the Fourier transform:

$$
\vec{J}_{s}\left(\vec{r}^{\prime}, t\right)=\frac{1}{2 \pi} \int \vec{J}_{s}\left(\vec{r}^{\prime}, \omega\right) \exp (j \omega t) d \omega .
$$

Substituting (4) to (7), $\vec{J}_{s}$ could also be written as

$$
\vec{J}_{s}\left(\vec{r}^{\prime}, t\right)=\frac{1}{2 \pi} \int 2 \widehat{n} \times \vec{H}^{\text {inc }}\left(\vec{r}^{\prime}, \omega\right) \exp (j \omega t) d \omega .
$$

By taking IFT of the incident magnetic field $\vec{H}^{\text {inc }}\left(\vec{r}^{\prime}, \omega\right)$, one can obtain its formulation in time domain:

$$
\vec{H}^{\text {inc }}\left(\vec{r}^{\prime}, t\right)=\frac{1}{2 \pi} \int \vec{H}^{\text {inc }}\left(\vec{r}^{\prime}, \omega\right) \exp (j \omega t) d \omega .
$$

Based on (6), (8), and (9), the scattering field in the time domain is derived as

$$
\begin{aligned}
& \vec{E}_{s}(\vec{r}, t) \\
& \quad=\frac{z_{0}}{2 \pi r c} \iint_{s} \hat{r} \times\left[\widehat{r} \times\left(\hat{n} \times \frac{\partial}{\partial t} \vec{H}^{\text {inc }}\left(\vec{r}^{\prime}, t-\tau_{1}-\tau_{2}\right)\right)\right] d s^{\prime},
\end{aligned}
$$

where $\tau_{2}=\widehat{k}_{i} \cdot \vec{r}^{\prime} / c$ is time delay in the incident direction. For the derivation in detail, one can refer to [12]. From the equation above, integration over the whole scattering area only refers to the incident field with time delay $\left(\tau_{1}+\tau_{2}\right)$ in the lit region and is not related to interaction between the other points on the surface. For each small triangle facet in lit region, (10) is implemented to calculate scattered electric fields in time domain at the point $\vec{r}$. The wide-band RCS is obtained through carrying out FFT on transient response. And the normalized RCS in the far zone is defined as [17]

$$
\mathrm{RCS}=\lim _{R \rightarrow \infty} \frac{4 \pi R^{2}}{S} \frac{\left|\vec{E}^{s}\right|^{2}}{\left|\vec{E}^{i}\right|^{2}},
$$

where $S$ is the illuminated area of $2 \mathrm{D}$ rough surface.

\section{Numerical Results and Discussions}

3.1. Validation of TDPO. In this section, the TDPO method is utilized to investigate the problem of transient scattering from 2D Gaussian randomly rough surface. It is noted that rough surface with finite length is concerned, similar to that in [18]; no tapering or window is introduced. Here, Gaussian pulse is chosen as the incident source. The proposed method is employed to calculate wide-band scattering from rough surface in the specular and backward direction with different incident angles, respectively, and its accuracy against KA is verified by comparing the $\theta \theta$ and $\varphi \varphi$ polarized results. In this paper, the proposed TDPO method is exhibited by averaging 15 Monte Carlo realizations.

Geometry of the scattering problem is illustrated and defined in Figure 2, where an incident wave impinges on the surface in the direction of $\widehat{k}_{i}$, which makes angle $\theta_{i}$ relevant to the $z$-axis and $\phi_{i}$ relative to the $x$-axis. The scattered angle is $\theta_{s}$, and the scattered azimuthal is $\phi_{s}$. The polarization angle is defined as $\eta$.

Because TDPO is valid only within the high frequency approximation, the incident source is a modulated Gaussian pulse, which is written as follows:

$$
e_{i}(t)=-\cos (\omega t) \exp \left[-\frac{4 \pi\left(t-t_{0}\right)}{\tau^{2}}\right],
$$

where $\omega=2 \pi f_{0}$ is the modulation circular frequency, $f_{0}$ is the modulation frequency, $t_{0}$ is the time delay, and $\tau$ is the pulse width.

In order to ensure the validity of the algorithm presented in this paper, we firstly calculate the RCS of 2D PEC rough surface using the TDPO method and KA, respectively. The size of rough surface generated is $S=2.4 \mathrm{~m} \times 2.4 \mathrm{~m}$, the rms height is $\sigma=0.01 \mathrm{~m}$, and the correlation length is 


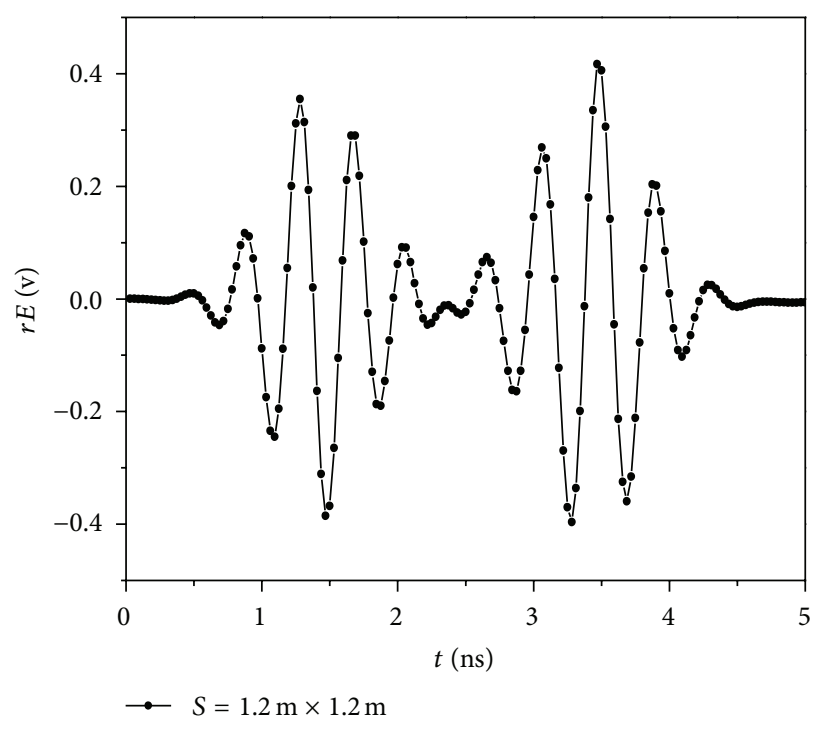

(a) Transient response $(S=1.2 \mathrm{~m} \times 1.2 \mathrm{~m})$

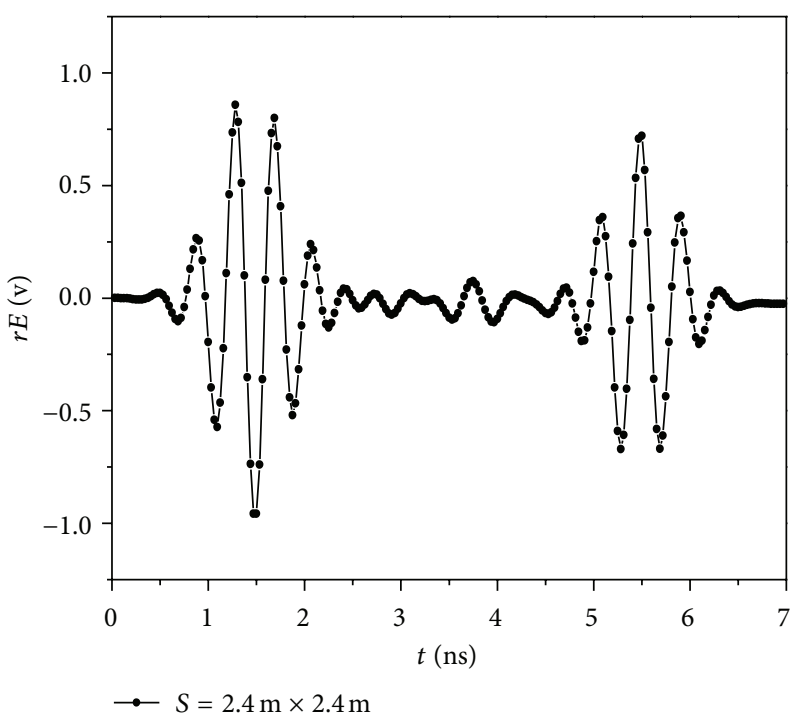

(b) Transient response $(S=2.4 \mathrm{~m} \times 2.4 \mathrm{~m})$

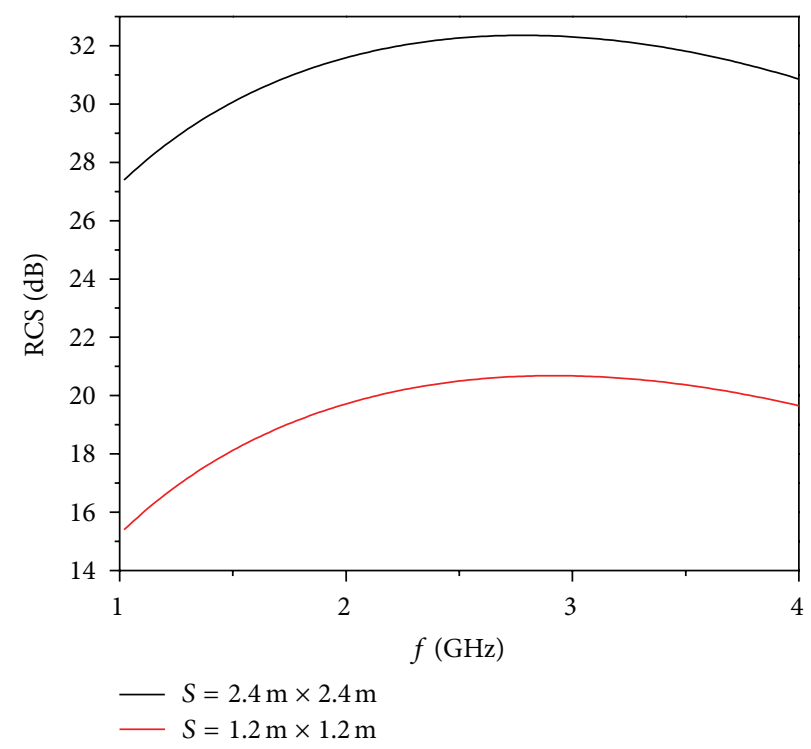

(c) Wide-band RCS

FIGURE 4: Scattering from 2D rough surface in specular direction for different sizes of rough surface.

$l_{x}=l_{y}=0.1 \mathrm{~m}$. The frequency band width and the pulse width of the modulated Gaussian pulse are $f=1 \sim 4 \mathrm{GHz}$ and $\tau=1.875 \mathrm{~ns}$, respectively. In Figure 3(a), the incident angle is $\theta_{i}=30^{\circ}$, the azimuth angle is $\phi_{i}=0^{\circ}$, and the polarization angle is $\eta=0^{\circ}$. In Figure 3(b), the incident parameters are set to $\theta_{i}=45^{\circ}, \phi_{i}=0^{\circ}$, and $\eta=90^{\circ}$.

Figure 3(a) compares the analysis results obtained by TDPO and those by KA in the backward directions with $\theta \theta$ polarization. Figure 3(b) presents the results of $\varphi \varphi$ polarization in the specular direction using above two mentioned methods. It is obvious that the scattering results by TDPO are coincident with those of KA method, which verifies the validation of the proposed TDPO. In addition, the curve of RCS from the rough surface in the specular direction is much smoother than that in the backward direction.
3.2. Discussion on the Results of EM Scattering from Rough Surface. In this section, the proposed TDPO method is employed to analyze rough surface with different sizes and different scale of rough surface in terms of correlation length and rms height both in the time and frequency domain. Moreover, the backscattering results for different incident angles are discussed.

In Figure 4, the scattering results from 2D PEC rough surface in the specular direction are shown for $\varphi \varphi$ polarization discussed with different sizes of surface $(S=1.2 \mathrm{~m} \times 1.2 \mathrm{~m}$, $S=2.4 \mathrm{~m} \times 2.4 \mathrm{~m}$ ), where the rms height and the correlation length are $\sigma=0.01 \mathrm{~m}$ and $l_{x}=l_{y}=0.1 \mathrm{~m}$, respectively. The incident angle is $\theta_{i}=30^{\circ}$, the incident azimuth angle is $\phi_{i}=0^{\circ}$, and the incident frequency is $f=1 \sim 4 \mathrm{GHz}$. Figures 4(a)-4(b) investigate the transient response rough surface, 


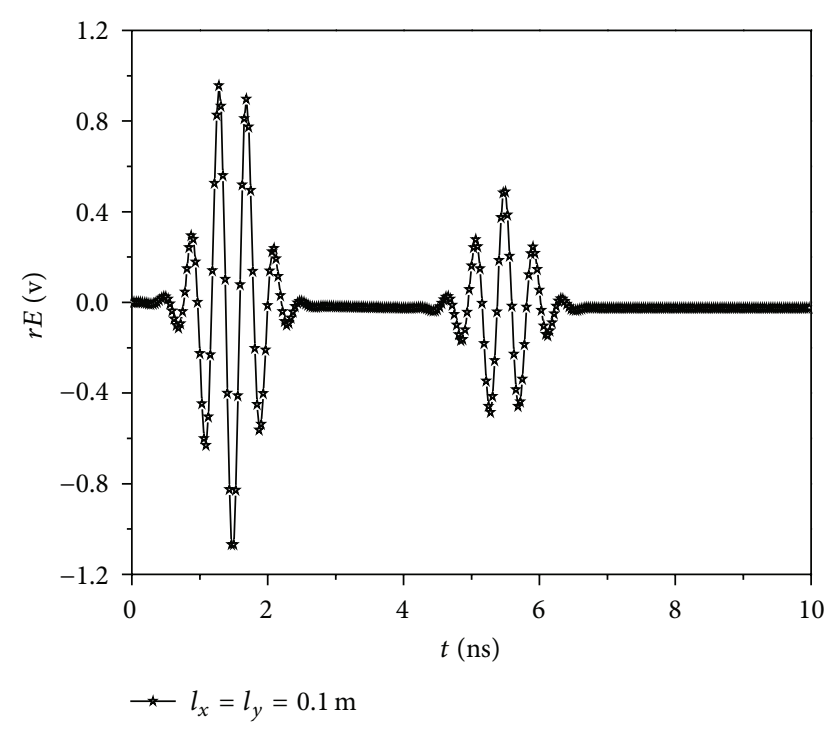

(a) Transient response $\left(l_{x}=l_{y}=0.1 \mathrm{~m}\right)$

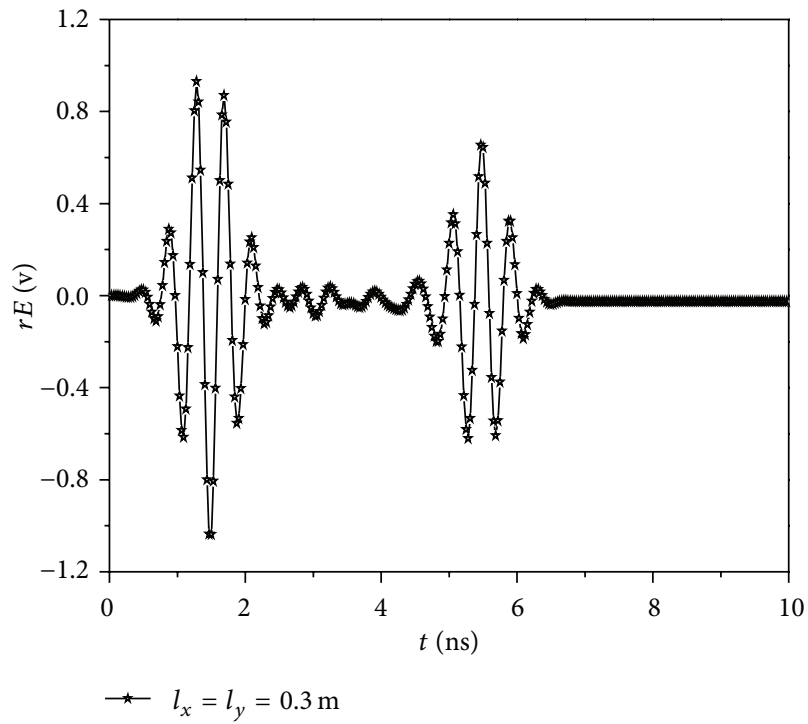

(c) Transient response $\left(l_{x}=l_{y}=0.3 \mathrm{~m}\right)$

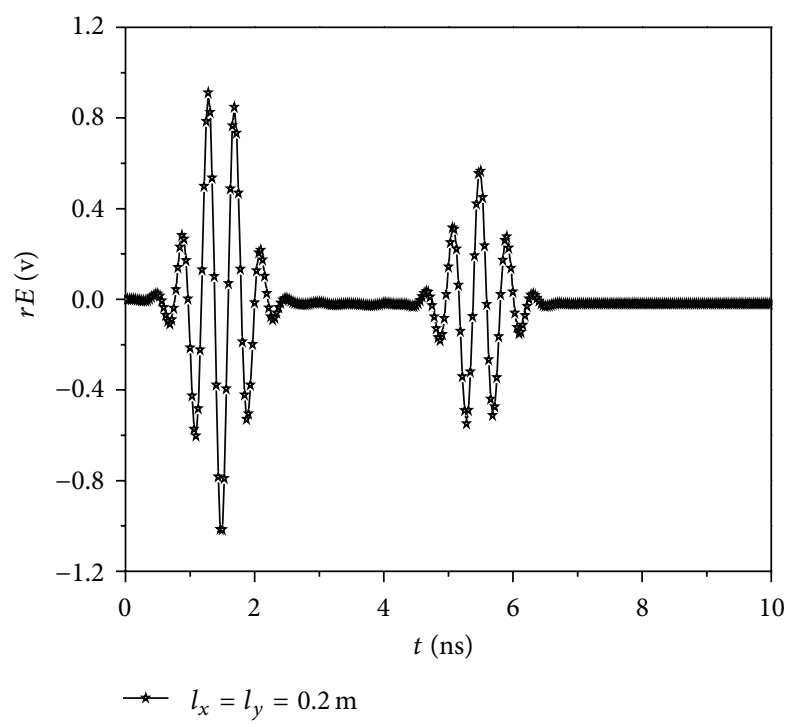

(b) Transient response $\left(l_{x}=l_{y}=0.2 \mathrm{~m}\right)$

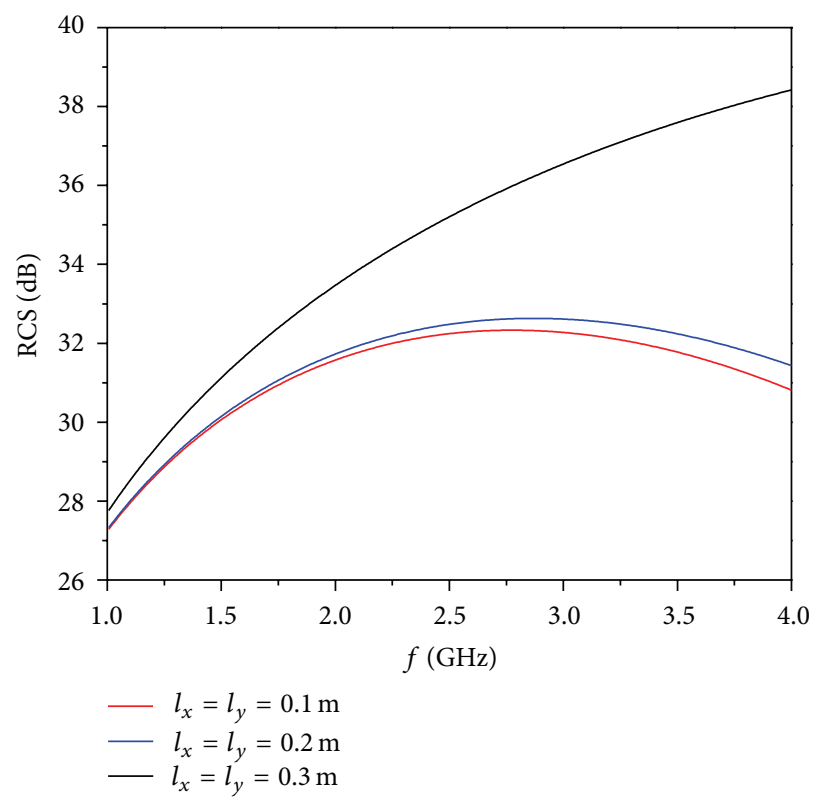

(d) Wide-band RCS

FIGURE 5: Scattering from the 2D PEC rough surface in the specular direction for different correlation lengths.

where it is obvious that the magnitude of the electric field gets larger when the size of the rough surface increases. We attribute this phenomenon to the fact that the scattering in the specular direction becomes stronger when the size of rough surface is larger. Figure 4(c) plots the wide-band RCS from the rough surface, where one can find that the RCS increases with increasing the surface size $S$ for the whole frequency bands. In addition, it is also seen that the variation tendency of the curve with parameter $S=1.2 \mathrm{~m} \times 1.2 \mathrm{~m}$ is similar to that with $S=2.4 \mathrm{~m} \times 2.4 \mathrm{~m}$. The reason for this phenomenon is that the parameters of the two kinds of rough surface are the same in terms of the correlation length and the rms height, which determine the profile of surface.
Figures 5(a)-5(c) plot the $\varphi \varphi$ polarized electric fields in the time domain from the rough surface in the specular direction, where the parameters of rough surface are $S=$ $2.4 \mathrm{~m} \times 2.4 \mathrm{~m}, \sigma=0.01 \mathrm{~m}$, and for the case of $l_{x}=l_{y}=0.1 \mathrm{~m}$, $l_{x}=l_{y}=0.2 \mathrm{~m}$ and $l_{x}=l_{y}=0.3 \mathrm{~m}$, respectively. The incident angle is $\theta_{i}=30^{\circ}$, and the incident azimuth angle is $\phi=0^{\circ}$. It is found that the first pulse signal has little difference, but it is obviously observed that the second pulse increases with increasing $l$.

Figure 5(d) shows the analysis result of wide-band characteristics through transforming transient response into frequency. It is obvious that RCS in the specular direction increases with increasing correlation length $l$, especially at 


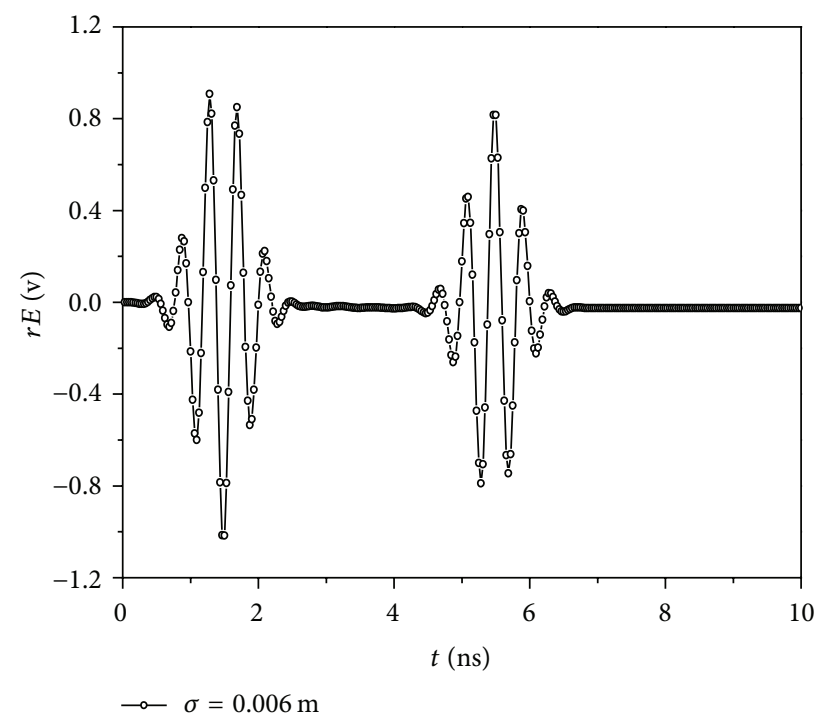

(a) Transient response $(\sigma=0.006 \mathrm{~m})$

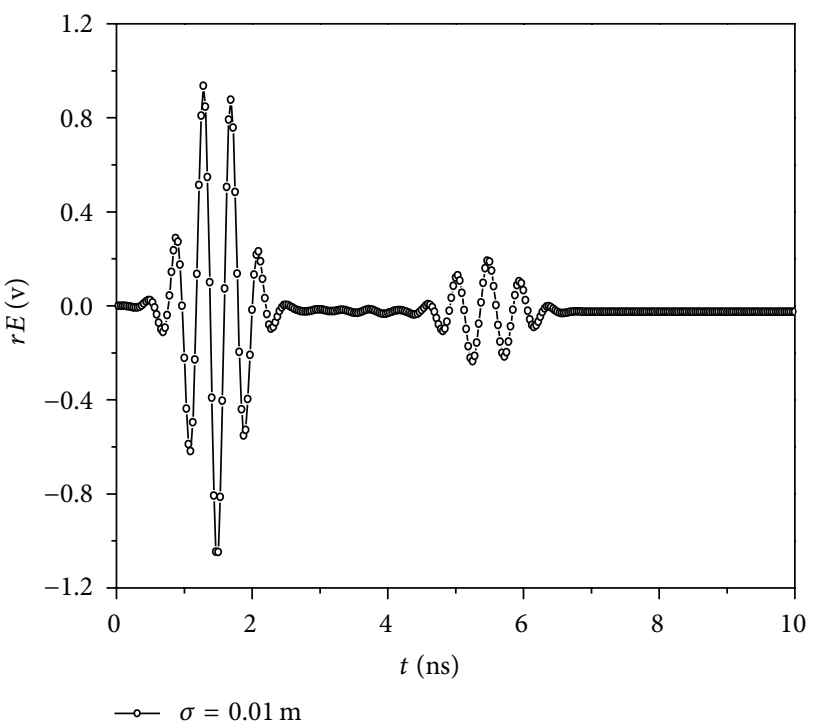

(c) Transient response $(\sigma=0.01 \mathrm{~m})$

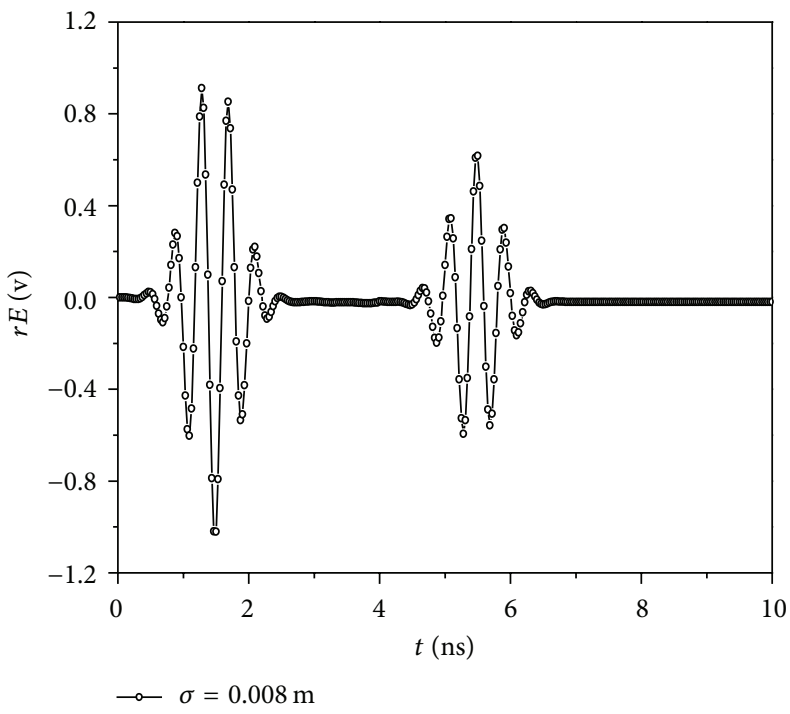

(b) Transient response $(\sigma=0.008 \mathrm{~m})$

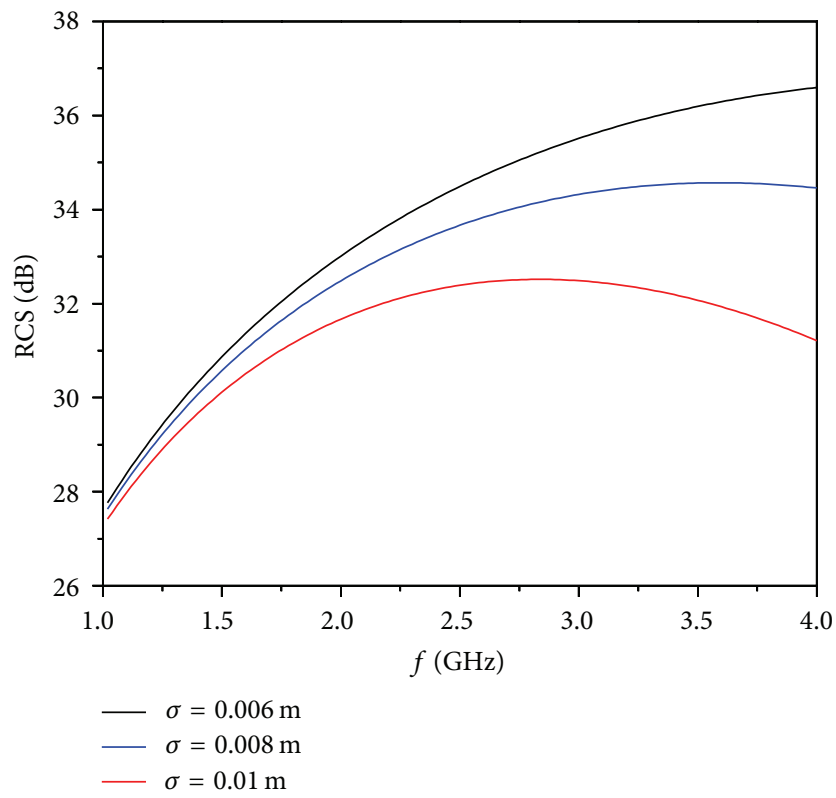

(d) Wide-band RCS

FIGURE 6: Scattering from the 2D PEC rough surface in the specular direction for different root square mean heights.

the high frequency band. The reason for this is that by keeping the rms height constant and by increasing the correlation length, the electromagnetic roughness is constant, but the rms slope decreases, which leads to an increase of the scattered energy in the coherent scattering direction.

The results of transient response and wide-band RCS from $2 \mathrm{D}$ rough surface in specular direction for different root square mean heights $\left(l_{x}=l_{y}=0.2 \mathrm{~m}, \sigma=0.006 \mathrm{~m}, 0.008 \mathrm{~m}\right.$, and $0.01 \mathrm{~m}$ ) are presented in Figure 6, where the size of rough surface is $S=2.4 \mathrm{~m} \times 2.4 \mathrm{~m}$, the incident angle is $\theta=30^{\circ}$, and the azimuth angle is $\phi_{i}=0^{\circ}$. The $\varphi \varphi$ polarization is discussed. Figures 6(a)-6(c) show the transient response from rough surface, where the magnitude of the second signal decreases with increasing rms height $\sigma$. In Figure 6(d), wide-band RCS decreases obviously with larger $\sigma$ over the whole frequency range; the primary reason for this is because the roughness increases with the increase of $\sigma$, which results in the decrease of the scattering in the specular direction with increasing of the surface roughness.

To further explore the important scattering characteristics of rough surface, the backscattering results $(\theta \theta$ polarization) for different incident angles are compared in Figure 7, where the size of Gaussian rough surface is $S=2.4 \mathrm{~m} \times 2.4 \mathrm{~m}$ and the rms height and the correlation length are $\sigma=0.01 \mathrm{~m}$ and $l_{x}=l_{y}=0.01 \mathrm{~m}$, respectively. The frequency band width of the Gaussian pulse is $f=1 \sim 4 \mathrm{GHz}$. 


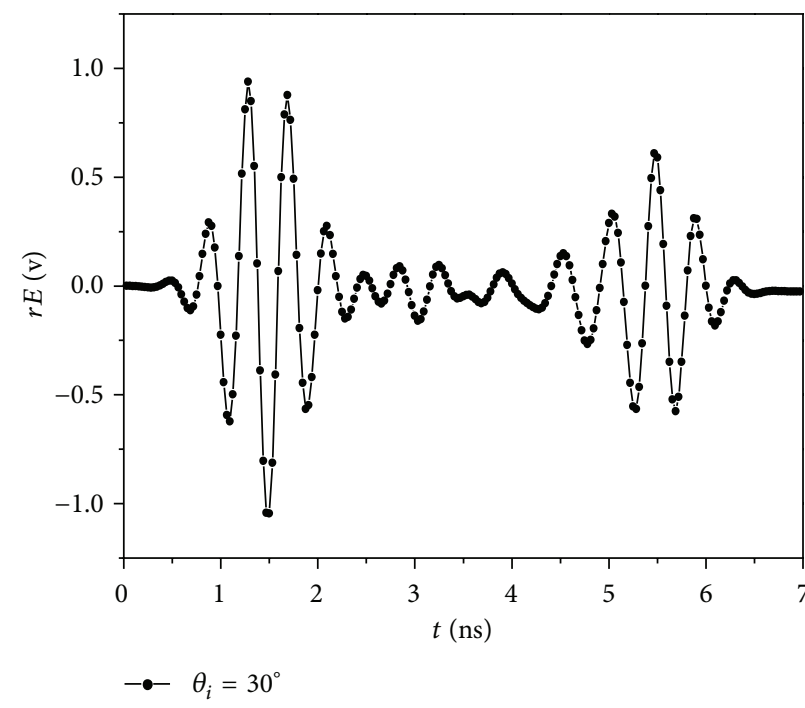

(a) Transient response $\left(\theta_{i}=30^{\circ}\right)$

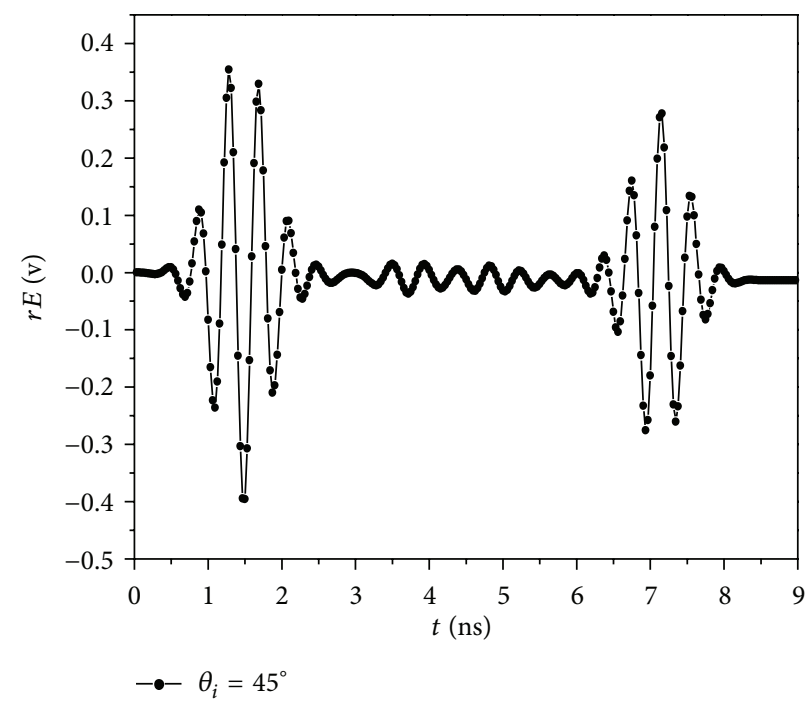

(b) Transient response $\left(\theta_{i}=45^{\circ}\right)$

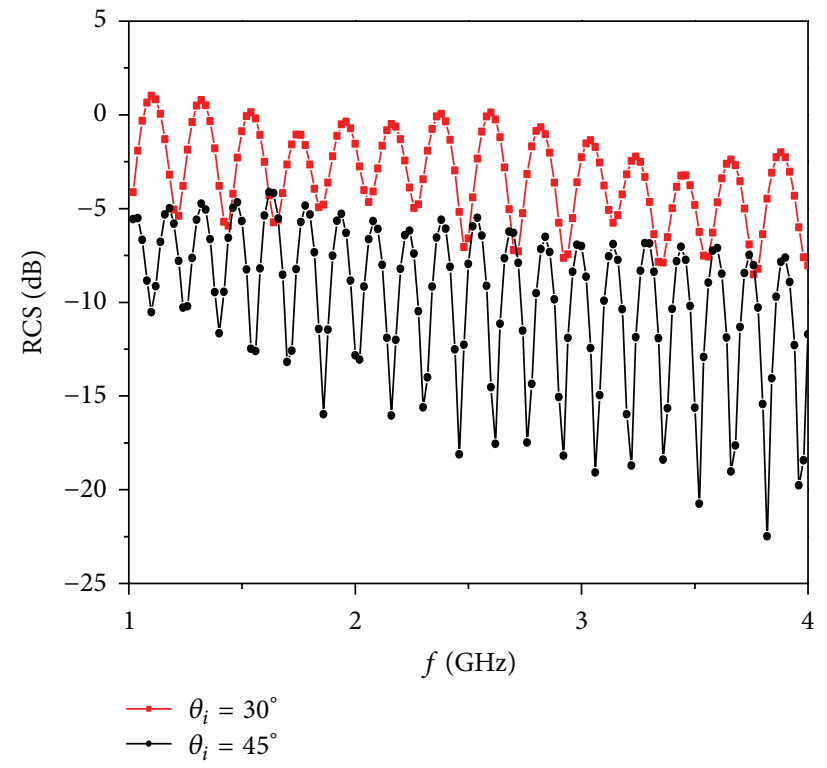

(c) Wide-band RCS in the backward direction

FIGURE 7: Backscattering from the 2D PEC rough surface for different incident angles.

Figures $7(a)-7(b)$ illustrate the backscattered electric fields in time domain, where the magnitude of electric field is visually smaller with increasing incident angles. The wideband RCS of 2D PEC rough surface is depicted in Figure 7(c). One can observe that the RCS becomes smaller with the increase of the incident angles over almost all the frequency band of $1-4 \mathrm{GHz}$, which is caused by the fact that the smaller angle leads to stronger scattering in the backward direction.

\section{Conclusion}

In this paper, a time-domain high frequency TDPO method is presented and adopted to investigate the scattering characteristics of 2D PEC Gaussian rough surface. Firstly, the wide-band RCS from 2D rough surface in both backward and specular directions calculated by the TDPO method are compared with the results obtained by the KA method. Good agreement is achieved for the two cases, which verifies the validation of the presented TDPO method. Then, the scattering characteristics of rough surface with different size are analyzed. The transient response in time domain and the wide-band RCS obtained by FFT in frequency domain are presented to examine the effect of the correlation lengths and rms height on scattering properties in detail. Finally, we will put our focus on investigating the dielectric and lossy surfaces by employing MECA [19] in the time domain (TDMECA). Furthermore, the backscattering from rough surface is present and analyzed for the different incident angles. Similar to KA method [20], the TDPO method presented in this paper is invalid for the case of larger 
incident angle or the larger roughness due to the neglect of the multiple scattering. It should be pointed out that the future investigation on this topic will include the scattering from $2 \mathrm{D}$ conducting rough surface at low grazing angle illumination with larger roughness and scattering from the dielectric rough surfaces.

\section{Acknowledgments}

This work was supported by the National Science Foundation for Distinguished Young Scholars of China (Grant no. 61225002), the Specialized Research Fund for the Doctoral Program of Higher Education (Grant no. 20100203110016), and the Fundamental Research Funds for the Central Universities (Grant no. K50510070001).

\section{References}

[1] D. Holliday, "Resolution of a controversy surrounding the Kirchhoff approach and the small perturbation method in rough surface scattering theory," IEEE Transactions on Antennas and Propagation, vol. 35, no. 1, pp. 120-122, 1987.

[2] S. O. Rice, "Reflection of electromagnetic waves from slightly rough surfaces," in Theory of Electromagnetic Waves, M. Kline, Ed., pp. 351-378, Wiley, New York, NY, USA, 1951.

[3] G. Berginc, "Small-slope approximation method: a further study of vector wave scattering from two-dimensional surfaces and comparison with experimental data," Progress in Electromagnetics Research, vol. 37, pp. 251-287, 2002.

[4] S. L. Durden and J. F. Vesecky, "Numerical study of the separation wavenumber in the two-scale scattering approximation," IEEE Transactions on Geoscience and Remote Sensing, vol. 28, no. 2, pp. 271-272, 1990.

[5] R. M. Axline and A. K. Fung, "Numerical computations of scattering from a perfectly conducting random surface," IEEE Transactions on Antennas and Propagation, vol. 26, no. 3, pp. 482-488, 1978.

[6] C. H. Chan, S. H. Lou, L. Tsang, and J. A. Kong, "Electromagnetic scattering of waves by random rough surface: a finitedifference time-domain approach," Microwave and Optical Technology Letters, vol. 4, no. 9, pp. 355-359, 1991.

[7] J. Li, L.-X. Guo, and H. Zeng, "FDTD investigation on bistatic scattering from two-dimensional rough surface with UPML absorbing condition," Waves in Random and Complex Media, vol. 19, no. 3, pp. 418-429, 2009.

[8] R. R. Lentz, "A numerical study of electromagnetic scattering from ocean-like surfaces," Radio Science, vol. 9, no. 12, pp. 11391146, 1974.

[9] R. T. Marchand, "On the use of finite surfaces in the numerical prediction of rough surface scattering," IEEE Transactions on Antennas and Propagation, vol. 47, no. 4, pp. 600-604, 1999.

[10] S. H. Lou, L. Tsang, and C. H. Chan, "Application of the finite element method to Monte Carlo simulations of scattering of waves by random rough surfaces: penetrable case," Waves in Random Media, vol. 1, no. 4, article 006, pp. 287-307, 1991.

[11] A. Iodice, "Forward-backward method for scattering from dielectric rough surfaces," IEEE Transactions on Antennas and Propagation, vol. 50, no. 7, pp. 901-911, 2002.

[12] V. Jandhyala, E. Michielssen, S. Balasubramaniam, and W. C. Chew, "A combined steepest descent-fast multipole algorithm for the fast analysis of three-dimensional scattering by rough surfaces," IEEE Transactions on Geoscience and Remote Sensing, vol. 36, no. 3, pp. 738-748, 1998.

[13] E.-Y. Sun and W. V. T. Rusch, "Time-domain physical-optics," IEEE Transactions on Antennas and Propagation, vol. 42, no. 1, pp. 9-15, 1994.

[14] L.-X. Yang, D.-B. Ge, and B. Wei, "FDTD/TDPO hybrid approach for analysis of the EM scattering of combinative objects," Progress in Electromagnetics Research, vol. 76, pp. 275284, 2007.

[15] L. Tsang and J. A. Kong, Scattering of Electromagnetic WavesNumerical Simulations, Wiley, New York, NY, USA, 2000.

[16] C. Scott, Modern Methods of Reflector Antenna Analysis and Design, Artech H, Boston, Mass, USA, 1990.

[17] J. Li, L.-X. Guo, and H. Zeng, "FDTD method investigation on the polarimetric scattering from 2-D rough surface," Progress in Electromagnetics Research, vol. 101, pp. 173-188, 2010.

[18] J. Li, B. Wei, Q. He, L. Guo, and D. Ge, “Time-domain iterative physical optics method for analysis of EM scattering from the target half buried in rough surface: PEC case," Progress in Electromagnetics Research, vol. 121, pp. 391-408, 2011.

[19] J. G. Meana, J. Á. Martínez-Lorenzo, F. Las-Heras, and C. Rappaport, "Wave scattering by dielectric and lossy materials using the Modified Equivalent Current Approximation (MECA)," IEEE Transactions on Antennas and Propagation, vol. 58, no. 11, pp. 3757-3761, 2010.

[20] A. Collaro, G. Franceschetti, M. Migliaccio, and D. Riccio, "Gaussian rough surfaces and Kirchhoff approximation," IEEE Transactions on Antennas and Propagation, vol. 47, no. 2, pp. 392-398, 1999. 

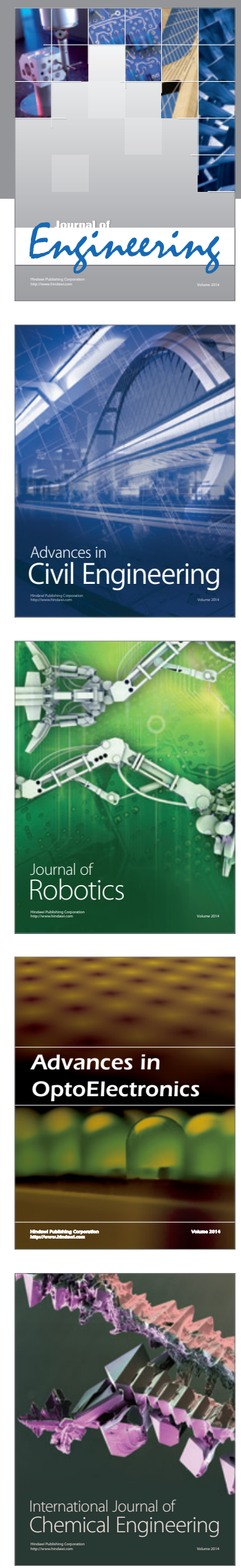

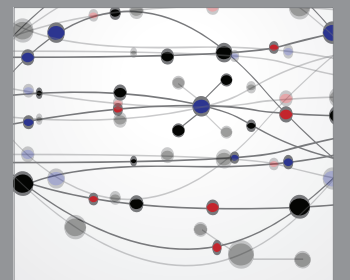

The Scientific World Journal
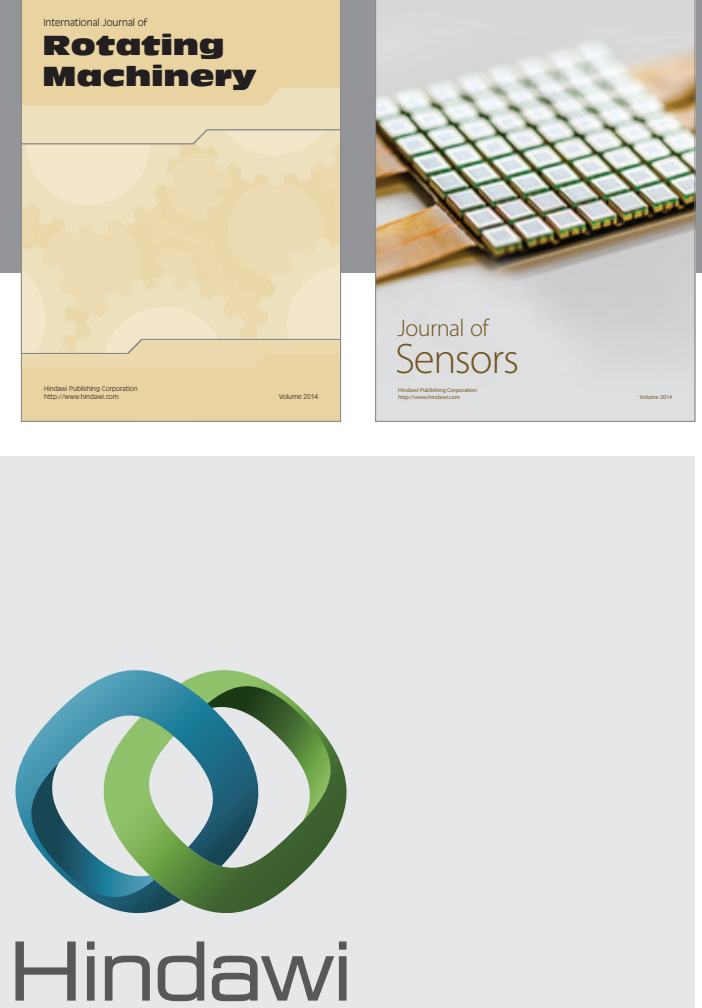

Submit your manuscripts at http://www.hindawi.com
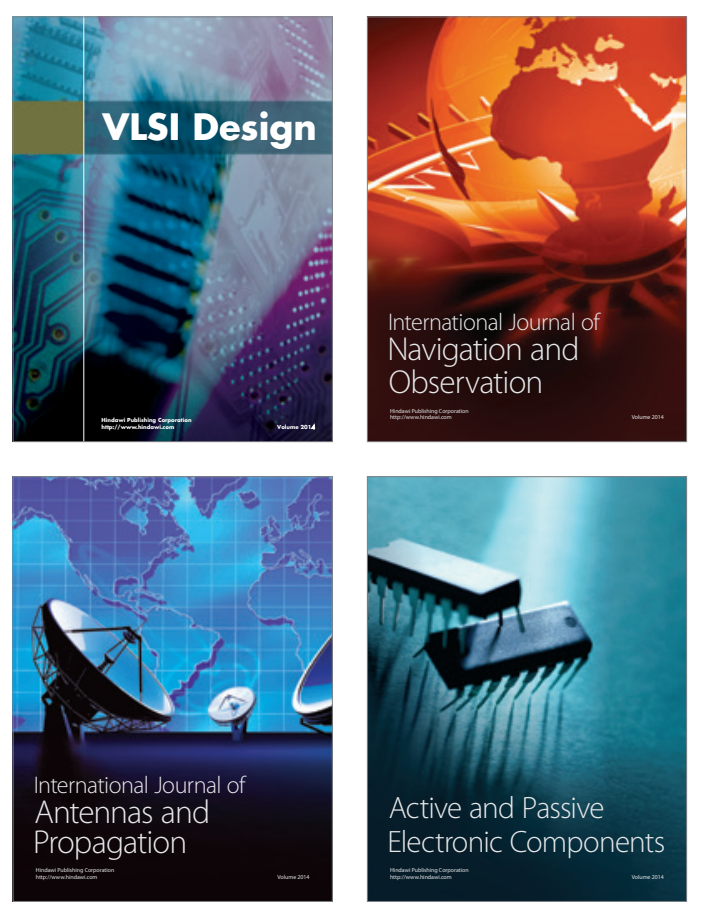
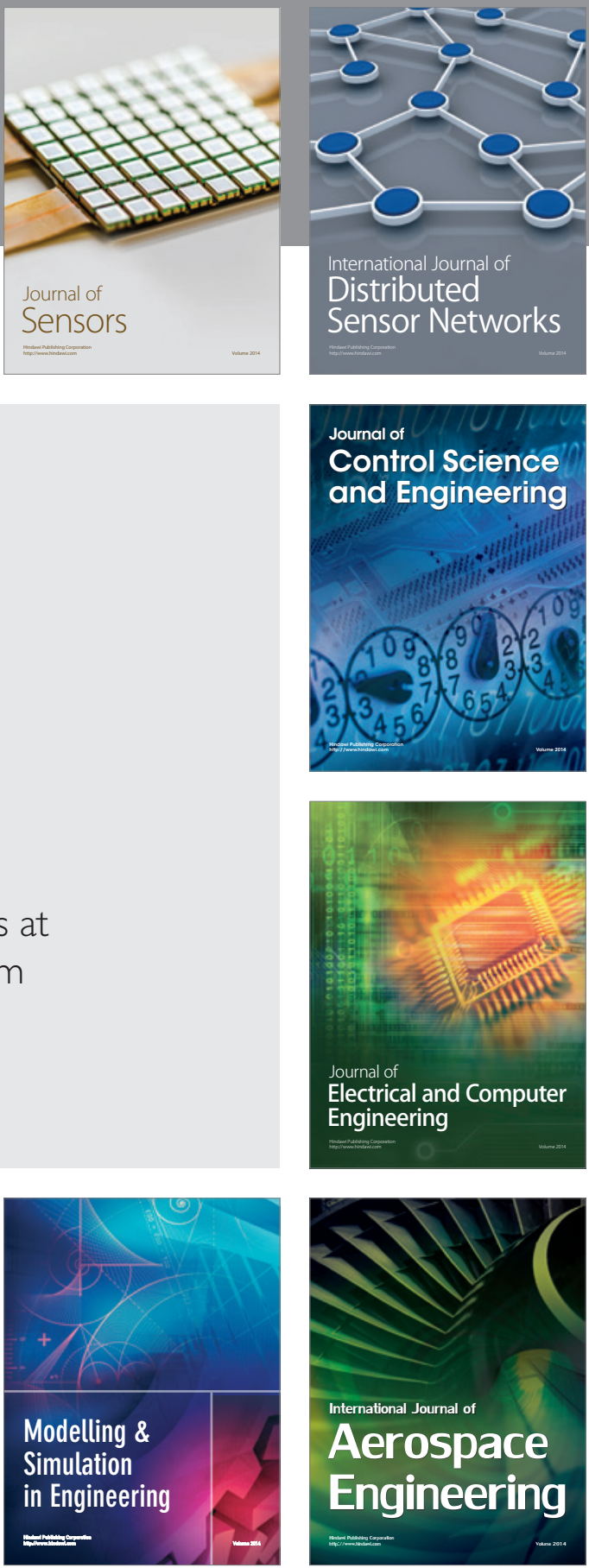

Journal of

Control Science

and Engineering
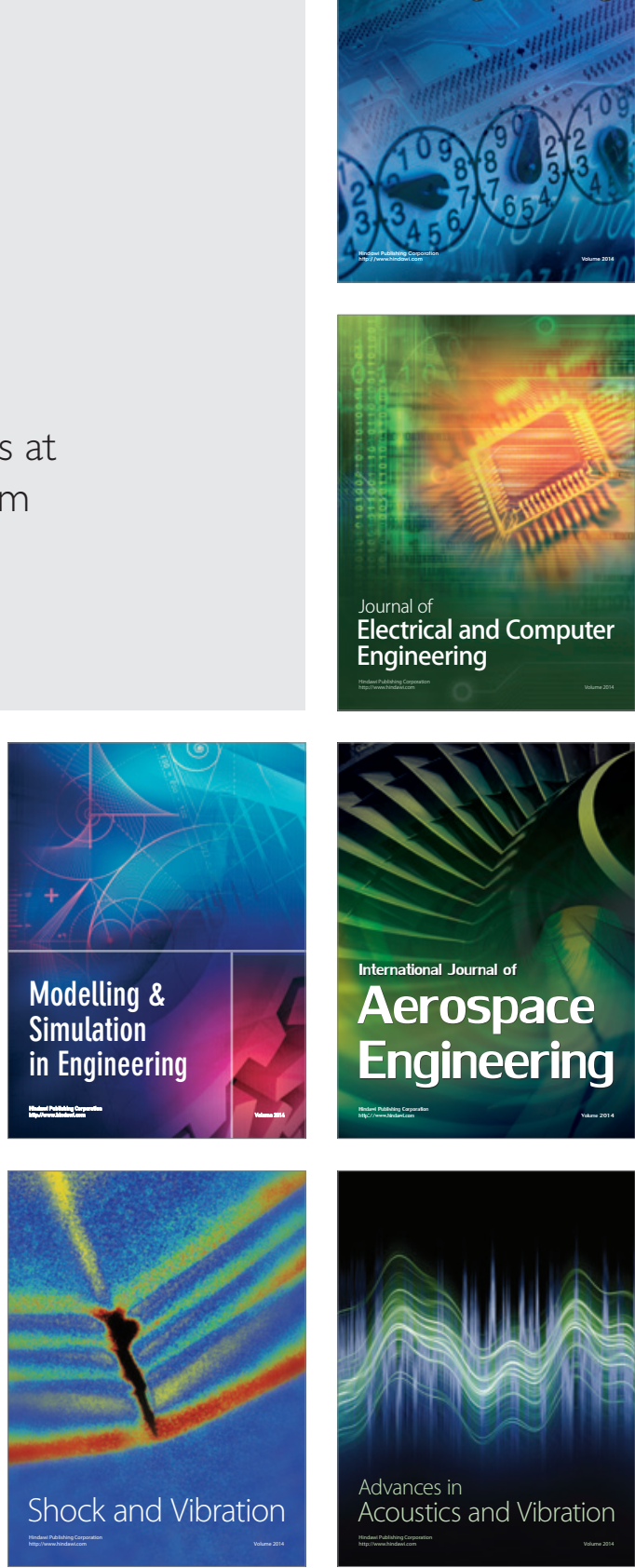